\title{
Reduced Complications after Arterial Reconnection in a Rat Model of Orthotopic Liver Transplantation
}

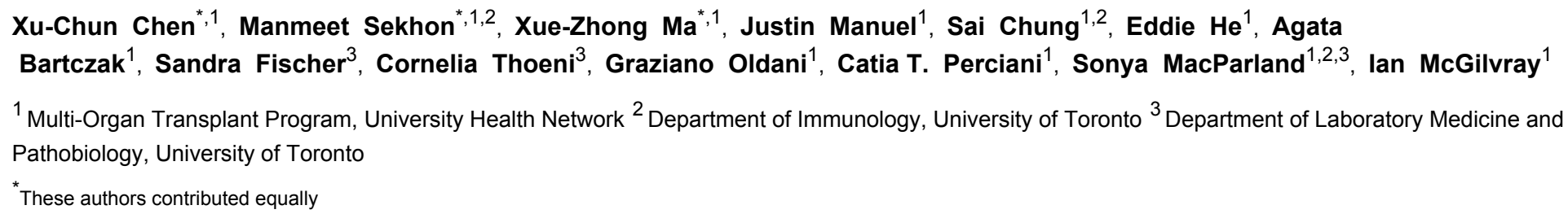

\section{Corresponding Authors}

Sonya MacParland

s.macparland@utoronto.ca

Ian McGilvray

ian.macgilvray@uhn.ca

\section{Citation}

Chen, X.C., Sekhon, M., Ma, X.Z.,

Manuel, J., Chung, S., He, E.,

Bartczak, A., Fischer, S., Thoeni, C.,

Oldani, G., Perciani, C.T.,

MacParland, S., McGilvray, I. Reduced

Complications after Arterial

Reconnection in a Rat Model of

Orthotopic Liver Transplantation. J. Vis.

Exp. (165), e60628, doi:10.3791/60628

(2020).

\section{Date Published}

November 7, 2020

DOI

$10.3791 / 60628$

URL

jove.com/video/60628

\section{Abstract}

The rat orthotopic liver transplantation (OLT) model is a powerful tool to study acute and chronic rejection. However, it is not a complete representation of human liver transplantation due to the absence of arterial reconnection. Described here is a modified transplantation procedure that includes the incorporation of hepatic artery (HA) reconnection, leading to a marked improvement in transplant outcomes. With a mean anhepatic time of $12 \mathrm{~min}$ and $14 \mathrm{~s}$, HA reconnection results in improved perfusion of the transplanted liver and an increase in long-term recipient survival from $37.5 \%$ to $88.2 \%$. This protocol includes the use of 3D-printed cuffs and holders to connect the portal vein and infrahepatic inferior vena cava. It can be implemented for studying multiple aspects of liver transplantation, from immune response and infection to technical aspects of the procedure. By incorporating a simple and practical method for arterial reconnection using a microvascular technique, this modified rat OLT protocol closely mimics aspects of human liver transplantation and will serve as a valuable and clinically relevant research model.

\section{Introduction}

The global burden of liver disease continues to increase, with a $30 \%$ rise in liver disease-related deaths from 2005 to $2013^{1,2}$. Liver transplantation is often the only recourse for patients with end-stage liver disease. The liver is the second most frequently transplanted solid organ, and the number of liver transplants performed globally increased by $7.25 \%$ from 2015 to $2016^{1,2}$. Despite its prevalence, post-transplantation survival rates have become stagnated ${ }^{3,4,5}$. The 15 year 
patient survival rate is reported to be $53 \%$, and the 20 year patient survival rate may be as low as $21 \%^{3,5}$. While there are exciting new immunobiology initiatives that may lead to new treatments and improved clinical outcomes, there is not yet a reliable small animal model in which to test them.

The rat OLT model has been widely used in the investigation of liver transplantation, including rejection $6,7,8,9,10$, immune tolerance $^{11}$, transplant ischemia-reperfusion injury ${ }^{12}$, immunosuppression ${ }^{13}$, and biliary tree injury ${ }^{14,15,16,17}$. However, a disadvantage of the model in its current form is its high post-operative morbidity and mortality ${ }^{18,19}$. This is a serious drawback that is at odds with the human operation, and it compromises the ability to draw clinically relevant conclusions from the model ${ }^{20}$.

Additionally, a large proportion of this morbidity can be attributed to an absent or imperfect hepatic artery (HA) reconnection ${ }^{18}$. Although a critical step in human liver transplantation, technical difficulties tend to compromise HA reconnection in the rat OLT model. As a result, bile duct (BD) anastomosis is tenuous and results in high rates of bile leakage and $\mathrm{BD}$ necrosis ${ }^{21}$. Beyond the high incidence of biliary complications ${ }^{22}$, an absence of arterial inflow alters the physiology of the graft liver posttransplantation $^{23}$, with hypoxia in the donor liver graft ${ }^{24}$ and liver damage observed in inflamed lobes ${ }^{19,25,26}$. Rat OLT without arterial reconnection also tends to promote fibrosis $^{27}$. The rat OLT protocol described below addresses these issues by incorporating a simple $\mathrm{HA}$ reconstruction step with a previously published rat OLT method ${ }^{28}$, resulting in preservation of liver parenchyma and improved survival rates.

Liver transplantation has three phases: (1) extraction of the liver graft from the donor, (2) preparation of the donor liver graft, and (3) replacement of the recipient liver with the liver graft. The procedure involves the manipulation of five anatomical structures: the suprahepatic inferior vena cava $(\mathrm{SHVC})$, portal vein $(\mathrm{PV})$, infrahepatic inferior vena cava (IHVC), hepatic artery (HA), and bile duct (BD).

OLT in the rat was first introduced by Lee et al. using microsuture anastomosis of the SHVC, PV and IHVC, and a pull-through technique for the $B D^{29}$. This model was later improved through use of the two-cuff technique in $1979^{30}$. Since then, several alternative techniques have been proposed, with the majority focusing on venous anastomosis and using a two-cuff technique with a few modifications ${ }^{31}$. Although HA anastomosis has been described previously in the rat OLT model using techniques such as microsuture, cuff, and intraluminal sleeves $26,31,32,33,34$, these techniques often require highly trained microsurgical skills, significantly alter rat physiology, and are hampered by thrombosis and/or biliary complications ${ }^{27,35}$.

Furthermore, the choice of surgical procedure can also influence the anhepatic time (time from PV clamping to reperfusion of graft through the reconstituted $\mathrm{PV}$ ), which is critical for the success of rat liver transplantation. Specifically, high survival rates are observed with anhepatic times of $15-20 \mathrm{~min}^{36}$, and $30 \mathrm{~min}$ is the upper limit for success ${ }^{37,38}$. Therefore, the goal of this method is to implement a less invasive and more easily adoptable surgical rat OLT model that is able to reconnect the hepatic artery, promote efficient perfusion of the transplanted liver, maintain flow to the recipient bile duct, and preserve the recipient's physiological condition.

Detailed here are all the steps of this revised protocol, including the manipulation of the celiac trunk of the donor liver as well as the use of 1) a $1.5 \mathrm{~mm}$ stent to perform an extraluminal sleeve connection with the recipient proper HA, 
2) a running suture for the SHVC reconstruction, 3) two 3D-

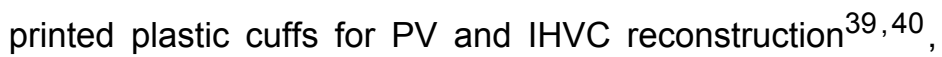
4) a microvascular sleeve reconnection for the $H A^{18,27,41}$ and 5) a previously described BD stenting technique ${ }^{28}$. Two additional steps are also included: a cold flush via the PV, and an antibiotic regimen that is based on previous findings ${ }^{17}$. This optimized OLT protocol minimizes perioperative complications and morbidity and more closely models the surgical operating procedure employed in human liver transplantation.

\section{Protocol}

The study was performed according to the guidelines of rodent handling and surgery, and the study protocol was approved by the University Health Network Animal Care Committee (UHN AUP \#: 5840.3) and follows the guidelines of the Canadian Council of Animal Care. The study uses male Lewis rats (strain LEW/SsNHsd), 12-14 weeks old, weighing between $250-300 \mathrm{~g}$.

\section{Equipment set-up}

1. Hold a $31 \mathrm{G}$ sharp tip with a needle holder and create a blunt L-shaped injector by flexing the tip repeatedly back and forth until the tip snaps off. Using a flat metal file, blunt and smooth the end of the injector.

1. Cut the portal vein (PV) and infrahepatic inferior vena cava (IHVC) cuffs from the 3D-printed base with a scalpel (Supplementary Material 1, Supplementary Material 2, Figure 1, Supplementary Figure 1).

NOTE: A 3D design software is used to design the cuffs and holders, which are printed on a 3D printer (Table of Materials) using autoclavable resin 39,40 (specifications for all 3D-printed material included in the Supplementary Material 1-10).

2. Use a new scalpel to cut the $22 \mathrm{G}$ catheter into a double-sided slanted tube (3.5 $\mathrm{mm}$ in length). Using the scalpel, gently etch lines onto the surface of the bile duct (BD) stent (do not cut through the wall of the tube). These etchings will prevent ties from slipping during the procedure.

3. Use a new scalpel to cut the $24 \mathrm{G}$ catheter into a one-sided slanted-edge tube $(2.0 \mathrm{~mm}$ in length), and create several scratches on the surface of the new arterial stent.

NOTE: Prevent narrowing or occlusion of the lumen of the BD stent by avoiding the application of pressure to the stent. If the stent is narrowed or occluded, the recipient's survival will be compromised by biliary obstruction.

\section{Donor operation}

1. Set a heat pad to $37{ }^{\circ} \mathrm{C}$ and place it underneath the surgical platform. Turn on the temperature monitor so that the rat's core temperature can be monitored via rectal probe. Set up the isoflurane anaesthesia apparatus.

NOTE: During surgery, monitor the depth of anesthesia by noting the breathing rate, heart rate, coloration of the organs/mucous membranes, and presence of any pedal withdrawal reflexes.

2. Arrange the surgically draped workspace with all necessary instruments and materials (i.e., scissors, forceps, gauze, heparin, retractors, midsection pad, cotton tips, 4-0 silk, 7-0 silk, 8-0 non-absorbable sterile suture, and 10-0 non absorbable monofilament suture) placed conveniently at the sides of the surgical platform. 
3. Arrange the workstation with all solutions, including Ringer's lactate solution and 300 IU of sodium heparin (see Table of Materials).

4. Weigh the animal. Anesthetize the donor rat by placing it in the anaesthetic chamber with $5 \%$ isoflurane, $5 \mathrm{~L} / \mathrm{min}$ air flow, and $70 \% \mathrm{FiO}_{2}$ for induction. When the rat loses consciousness, decrease anaesthesia to $3 \%$ isoflurane, $0.5 \mathrm{~L} / \mathrm{min}$ air flow, and $70 \% \mathrm{FiO}_{2}$. Check for the lack of pedal response by pinching the toe.

5. Prepare the skin of the abdomen. Using an electric shaver, remove the fur from the ventral side. Attentively observe the donor's respiration rate until it attains a stable and deep rate.

1. Lay the surgically draped rat so that its ventral side is facing the ceiling. Place the nose in an anesthesia scavenger with $3 \%$ isoflurane, $0.5 \mathrm{~L} / \mathrm{min}$ air flow, and $70 \% \mathrm{FiO}_{2}$. Prepare the abdominal wall with povidoneiodine, working from the midline outward, followed by $70 \%$ ethanol.

6. Make an incision from the xiphoid process to the pubic symphysis using a round-tipped surgical scissor, then improve exposure with a bilateral transverse incision. Stop any bleeding from the abdominal wall using a bipolar electrosurgical unit for cautery. After the incision, decrease maintenance isoflurane to $2 \%, 0.5 \mathrm{~L} / \mathrm{min}$ air flow, and $70 \% \mathrm{FiO}_{2}$.

NOTE: Adjust the isoflurane vaporizer to achieve a respiration rate of approximately one breath per second and remember to regularly assess the depth of anesthesia throughout the course of the operation.

7. Place a 4-0 silk stitch in the xiphoid process and use the suture to retract the chest wall cephalad. Tape the silk thread to the top of the structure that holds the anesthesia scavenger in place. Hold the donor rat's body cavity open with 3D-printed retractors (see Supplementary Material 3) placed on the either side of the abdomen (retractors are held in place with rubber bands attached to magnets on the surgical platform).

NOTE: Mosquito forceps also can be used to grasp the xiphoid process and retract the cephalad. Fix the mosquito forceps in place using tape.

8. Use a nonwoven gauze sponge $(4 \mathrm{~cm} \times 4 \mathrm{~cm})$ dampened by Ringer's lactate solution to enclose the small and large intestines. Use a small, wet, nonwoven gauze sponge (2 $\mathrm{cm} \times 4 \mathrm{~cm}$ ) to gently cover the liver.

9. Place a small rolled gauze under the midsection to lift the abdomen and improve exposure of the suprahepatic inferior vena cava (SHVC).

10. Cut the falciform ligament. Separate the left diaphragmatic vein from the SHVC using micro-forceps. Ligate the left diaphragmatic vein with 7-0 silk, staying close to the SHVC.

NOTE: Use the small, wet, nonwoven gauze sponge, dampened with Ringer's lactate and placed on the liver, to gently retract the liver away from the xiphoid process and expose the left diaphragmatic vein.

11. Cut the left triangular and the gastro-hepatic ligaments with round-tipped scissors.

12. Expose the caudate lobe by carefully pulling back the left and middle lobes towards the xiphoid process using a small, wet, nonwoven gauze sponge. Release the ligament separating the caudate lobe from the rest of the liver with a round-tipped scissor.

13. Divide and separate the hepato-esophageal ligament using a bi-polar electrosurgical unit close to the esophagus. 
NOTE: Gently shift the small and large intestines to the left side of the abdominal cavity and cover them with wet, nonwoven gauze.

14. Dissect the retroperitoneum and fat covering the IHVC. Expose and isolate the IHVC down to the left renal vein.

15. Slightly displace the IHVC with a cotton swab to expose and then cauterize any small veins merging into the right side of the IHVC, using the bi-polar electrosurgical unit. Also cauterize any lumbar veins merging into the IHVC.

16. Divide the right suprarenal (adrenal) vein between two 7-0 silk ligatures, staying close to the IHVC. Free the liver from its posterior ligaments by cutting these under gentle traction.

17. Isolate the right renal vein from the right renal artery and from the neighboring tissue using a fine-tip cautery. Seal the right renal vein orifice with an 8-0 non absorbable sterile ligature.

18. Detach the fat covering the PV to locate the pyloric vein (right gastric vein) and the splenic vein at the points where they merge the PV. Ligate these veins with 7-0 silk, reinforcing the side closest to the PV with an 8-0 non absorbable sterile suture stitch. Divide the veins between the ties.

NOTE: Expose the PV by using small, wet gauze to draw back the duodenum. The insertion of the cuff is easier if fat is detached from the PV, which also prevents stenosis of the PV cuff.

19. Inject $300 \mathrm{IU}$ of heparin sodium into the IHVC, diluted to 1 $\mathrm{mL}$ of normal saline, using a $1 \mathrm{~mL}$ syringe ( $31 \mathrm{G}$ needle).

20. Make an incision $5 \mathrm{~mm}$ below the BD bifurcation and insert the BD stent into the common BD. Secure the stent with a 7-0 silk ligature $1 \mathrm{~mm}$ above the incision. An extra tie can be made below the incision, which is $10 \mathrm{~mm}$ below the bifurcation. Once the stent is secured, cut the BD between these two ties.

21. Never clip the BD or proper hepatic artery (HA). Place a 10-0 non absorbable sterile surgical monofilament suture stitch at the 3 o'clock position in the BD at the incision as a marker to prevent twisting after reconnection.

22. Expose the proper HA and divide the gastroduodenal artery (GDA) between two 7-0 silk ligatures. Expose the left gastric artery, splenic artery, and celiac trunk. Tie the three arteries both distally and close to their take-offs.

23. Cut the left gastric artery, splenic artery, and celiac trunk between the artery ties. Slowly inject $20 \mathrm{~mL}$ of cold (4 ${ }^{\circ} \mathrm{C}$ ) Ringer's lactate solution into the PV, using a $20 \mathrm{~mL}$ syringe with a $21.5 \mathrm{G}$ needle. Cut the vena cava below the point at which the left renal vein merges with the IHVC to permit flush outflow.

NOTE: The needle should be kept as far as possible from the hilum. The donor liver cold perfusion should last between 1-2 min. While flushing the liver, use the other hand to squirt cold Ringer's lactate onto the liver surface.

24. Cut the PV trunk below the splenic vein after the flush. Cut the IHVC just above the left renal vein. Cut the SHVC directly adjacent to the diaphragm.

25. Cut the ligaments and connective tissue between the liver and retroperitoneum.

NOTE: Ensure that there are sufficient lengths of the anterior and posterior SHVC walls to facilitate the upper caval anastomosis. It is crucial to cut immediately adjacent to the diaphragm to retain as much length as possible.

26. After the liver has been removed from the abdomen, quickly place it into a dish filled with $4{ }^{\circ} \mathrm{C}$ Ringer's lactate 
solution. Place the dish on top of an ice pad to maintain a cold temperature.

27. Discard the donor rat's remains, following institutional guidelines.

\section{Donor rat liver preparation ("back bench")}

1. Fill the cold Petri dish with a sufficient volume of 4 ${ }^{\circ} \mathrm{C}$ Ringer's lactate solution to submerge the donor rat liver. Carefully rotate the donor liver floating in the dish carefully so that the inferior surface faces upward. Put the cuffs for PV and IHVC (Supplementary Material 1 and Supplementary Material 2, respectively) into the dish.

2. Pull the PV through the PV cuff and fold the end of the vein over the cuff. Tie the PV securely around the cuff using 7-0 silk. Flush the PV with $10 \mathrm{~mL}$ of $4{ }^{\circ} \mathrm{C}$ Ringer's lactate solution.

3. Repeat step 3.2 with the IHVC, without the flush.

4. Remove the fat tissue around the celiac trunk. Form a larger arterial sleeve cuff by cutting open the bifurcation of the celiac trunk, splenic artery, and left gastric artery (Figure 2A).

NOTE: It is difficult to insert the arterial stent into the common HA. Stretch and straighten the artery out with forceps several times before inserting the stent. Make sure that the bevel of the stent faces upward and that the artery is not twisted (Figure 2B).

5. Put the $1.5 \mathrm{~mm}$ length $24 \mathrm{G}$ arterial stent into the donor common HA via the arterial cuff. Secure the stent with an 8-0 polypropylene ligature (Figure $\mathbf{2 C}$ ) and flush the stent with Ringer's lactate solution (Figure 2D).

6. Position a micro-clamp (4-6 $\mathrm{mm}$ in length) onto the proximal IHVC, which is intended to prevent blood loss after portal reperfusion and avoid air embolism.
7. Rotate the liver and expose its superior side. Insert two 8-0 polypropylene taper point sutures on the lateral and medial edges of the SHVC.

8. Keep the liver at $4{ }^{\circ} \mathrm{C}$ so it is ready for transplantation into the donor.

\section{Recipient operation}

1. Refer to the donor operation section above and repeat steps 2.1-2.4.

NOTE: Male Lewis rats aged 12-14 weeks old are used here, weighing 5-20 g heavier than the donors. During the surgery, monitor the depth of anesthesia by noting the breathing rate, heart rate, coloration of the organs/mucous membranes, and presence of any pedal withdrawal reflexes.

2. Lay the surgically draped rat with its ventral side facing upwards. Place the nose in the anesthesia scavenger for isoflurane inhalation. Moisten the eyes with opthalamic lubricant. Prepare the abdominal wall with providoneiodine first, then with $70 \%$ ethanol.

3. Inject $5 \mathrm{~mL}$ of Ringer's lactate solution subcutaneously on both lower sides of the ventral abdominal wall. Use a surgical assistant's aide to inject $0.5 \mathrm{~mL}$ of $200 \mathrm{mg} / \mathrm{kg}$ piperacillin sodium intramuscularly into the left abdominal wall before laparotomy. In addition, administer $0.5 \mathrm{~mL}$ of $10 \mathrm{mg} / \mathrm{mL}$ bupivacaine subcutaneously into the right abdominal wall.

NOTE: Administer the same dose of piperacillin sodium $1 \mathrm{x} /$ day for 3 days post-operatively.

4. Prepare the abdominal wall again with providone-iodine first and then with $70 \%$ ethanol. Make a midline incision from the sternal xiphoid to $1 \mathrm{~cm}$ above the pubic symphysis. Decrease isoflurane to $2 \%, 0.5 \mathrm{~L} / \mathrm{min}$ air 
flow, and $\mathrm{FiO} 270 \%$ for maintenance of anesthesia after making the incision.

NOTE: Mosquito forceps can be used to grasp the xiphoid process and retract the cephalad. Fix the mosquito forceps in place using tape. The body cavity is kept open by 3D-printed retractors (see Supplementary Material 3) on both sides with rubber bands magnetically held to the surgical platform

5. Wrap the small and large intestines with a wet, nonwoven gauze sponge $(4 \mathrm{~cm} \times 4 \mathrm{~cm})$ dampened with Ringer's lactate solution. Use a small $(2 \mathrm{~cm} \times 4 \mathrm{~cm})$, wet, nonwoven gauze sponge dampened with Ringer's lactate solution to gently cover the liver.

6. Place a small 3D-printed support pad (back holder; see Supplementary Material 4) under the rat midsection to increase exposure of the SHVC by flexing the spin. This can safely be performed in rats and is executed by a surgeon assistant.

7. Cut the falciform ligament and use the small, wet nonwoven gauze sponge to gently retract the liver away the xiphoid process and expose the left diaphragmatic vein. Separate the left diaphragmatic vein from the SHVC using micro-forceps. Ligate the left diaphragmatic vein with 7-0 silk close to the diaphragm.

8. Cut the left triangular and the gastro-hepatic ligaments with round-tipped scissors.

9. Pull the left and middle lobes delicately toward the xiphoid process with a small, wet nonwoven gauze sponge to reveal the caudate lobe. Sever the ligament that separates the caudate lobe from the remainder of the liver.

10. Divide the hepato-esophageal ligament and coagulate any bleeding points with the bi-polar electrosurgical unit, staying close to the liver. Cut the ligaments at the posterior aspect of the liver.

11. Retract the small and large intestines carefully to the left side of the abdominal cavity and cover them with wet, nonwoven gauze.

12. Dissect the retroperitoneum and fat on the IHVC to expose and isolate the IHVC down to the right renal vein. Slightly displace the IHVC with a cotton swab and cauterize any small veins merging into the right side of the IHVC, using the bi-polar electrosurgical unit. Similarly, cauterize any lumbar veins entering the IHVC.

13. Divide the right suprarenal (adrenal) vein between two 7-0 silk ligatures. Free the liver from its posterior ligaments by cutting them under gentle traction.

14. Use small, wet gauze dampened with Ringer's lactate solution to retract the duodenum and expose the PV. Detach fat from the bifurcation of the PV and pyloric vein.

15. Divide the BD $0.5 \mathrm{~cm}$ below its hilar bifurcation and insert a BD stent into the distal common BD. Secure the stent in position with a 7-0 ligature about $0.2 \mathrm{~mm}$ below the incision. An extra tie can be placed above the incision, close to the bifurcation. Cut the BD close to liver but distal to the tie.

16. Separate the BD with forceps and avoid clipping the BD or proper HA. Place a 10-0 non absorbable monofilament (e.g., ethilon) stitch at the 3 o'clock position in the BD as a marker to prevent twisting after reconnection.

17. Expose the proper HA and the bifurcation of the common $H A$ and GDA. Expose the left $H A$, middle $H A$, and right HA. Tie the three arteries distal to the CHA bifurcation and cut the arteries close to the liver, above the ties.

18. Put a long thin piece of gauze behind the SHVC. 
19. Place a 3D-printed IHVC holder or "handle" (Cava 150g 2.1; see Supplementary Material 5) behind the IHVC, and sew the ends of the 3D-printed "handle" together using 10-0 non absorbable monofilament suture (Figure 3A).

20. Place a 3D-printed PV holder or "handle" (Porta 1.4.1see Supplementary Material 6) behind the PV, directly inferior to the liver, and sew the ends of the 3Dprinted "handle" together using 10-0 non absorbable monofilament suture.

21. Loosely tie a 7-0 silk ligature below both 3D-printed holders (IHVC and PV) (Figure 3A).

22. Clamp the IHVC just above the right renal vein, which should still be below the 3D-printed caval holder.

23. Clamp the PV just above the pyloric vein, which should be below the 3D-printed PV holder. Record the anhepatic time, which begins at this point. Decrease to $0.5 \%$ isoflurane, $0.5 \mathrm{~L} / \mathrm{min}$ air flow, and $70 \% \mathrm{FiO}_{2}$ for maintenance of anesthesia.

24. Flush $2 \mathrm{~mL}$ of $37{ }^{\circ} \mathrm{C}$ Ringer's lactate solution via the bifurcation of the PV using a $3 \mathrm{~mL}$ syringe with a $27 \mathrm{G}$ needle attached.

25. Clamp the SHVC above the liver with a Kitzmiller clamp. Cut below the same clamp, staying as close to the liver as possible.

26. Cut above the 3D-printed holders for both the PV and IHVC (Figure 3A). Remove the recipient's liver. Carefully orient the donor liver and position it in the recipient's body cavity in such a way that the upper caval anastomosis can be created.

27. Use an 8-0 polypropylene running suture to join the donor's SHVC with the recipient's SHVC near the diaphragm. First, place stay sutures of 8-0 polypropylene to the left and right aspects of the donor and recipient SHVC. Then, tie these down on the outside of the vein wall.

28. Use the left 8-0 polypropylene to stitch the posterior wall of the SHVC from left to right and tie to the right 8-0 polypropylene. Use the left 8-0 polypropylene to stitch the anterior wall of the SHVC anastomosis from left to right, leaving the last two-thirds of the suture line loose. Flush using $20 \mathrm{~mL}$ of Ringer's lactate in between the loose stitches while making sure to extract any air bubbles.

29. Tighten the loose stitches and make a tie on the outside of the SHVC. Cut the remaining 8-0 polypropylene suture. NOTE: The clip holds the recipient's SHVC in place, making it easier to sew the donor and recipient's SHVC together. Record the duration of SHVC anastomosis. At this point, the porta handles are attached to the holder arm apparatus (holder arm McGil + holder mini arm LAB + holder arm soft part 1.3; see Supplementary Material

\section{7, Supplementary Material 8, and Supplementary} Material 9, respectively), directly inferior to the liver. This apparatus is supported by a 3D-printed holder base (holder base 3.1; see Supplementary Material 10).

30. Insert the PV cuff (Supplementary Material 1) from the donor into the recipient PV and tighten the 7-0 silk tie. Flush the PV of the donor and recipient with Ringer's lactate solution warmed to $37^{\circ} \mathrm{C}$ before the connection.

31. Remove the atraumatic clamp from the SHVC (first), then the microvascular clip for the PV (second). Re-perfuse the liver with warm blood; at this point, the anhepatic phase time has ended. Record this time. 
32. Pour $10 \mathrm{~mL}$ of warm Ringer's lactate solution on top of the liver to warm. Remove the 3D-printed holders with roundtipped scissors (cut the securing stitch).

33. Insert the donor IHVC cuff (Supplementary Material 2) into the recipient IHVC and secure with a 7-0 silk tie. Remove the donor IHVC clip first, then the recipient's clip (Figure 3B). The cava is attached to the holder apparatus and holder base as described above,

34. Remove the 3D printer holders (porta and cava) with round-tipped scissors (cut the securing stitch; Figure $3 \mathrm{C}$ ), resulting in a connected IHVC (Figure 3D).

35. Carefully examine the area around the liver for any bleeding. Instil $3 \mathrm{~mL}$ of $37{ }^{\circ} \mathrm{C}$ Ringer's lactate solution inside the body cavity.

36. Arterial anastomosis: cut off the portion of the celiac trunk from the donor that extends beyond the stent.

37. Clamp the recipient's proper HA and cut off the tie at the end. Cut off any extra tissue surrounding the vessel (Figure 4A). With Ringer's lactate solution, flush the lumens of both donor and recipient vessel ends.

38. Pull the recipient proper HA into the sleeve of the donor HA stent to accomplish the HA anastomosis. Place a 10-0 ethilon through the left aspect of the (donor) HA, $2.5 \mathrm{~mm}$ above the distal orifice of the stent (from outside to inside), then out through the end of the stent, with 10-0 ethilon (4 $\mathrm{cm}$ length) guided by a curved needle (Figure 4B).

39. Transfix the recipient proper HA $0.5 \mathrm{~mm}$ below the vessel orifice, placing the stitch first (from inside to outside) to the left side of the vessel, then (from outside to inside) to the right side of the artery.

40. Place the suture through the right wall of the (donor) HA from the inside to outside, at a distance from the stent orifice identical to the original stitch. Pull up on the two ends of the 10-0 non absorbable monofilament, which will slip the recipient proper HA up and into the HA stent (Figure 4C).

NOTE: Observe for pumping of blood. One option is to cut the donor GDA to confirm that blood is pumping through the anastomosis. Make sure to re-tie the artery before moving onto the next step of the procedure if the GDA is severed.

41. Tie the 10-0 non absorbable monofilament with itself, over the donor HA (Figure 4D). The arterial anastomosis is now complete.

42. Biliary anastomosis: place a tie loosely around the recipient $B D$ and stent (Figure $5 A$ ), then remove the $B D$ stent. Flush the BD of both recipient and donor before the biliary connection is complete.

43. Insert the donor's BD stent into the recipient's bile duct (Figure 5B) and tighten the tie that was previously placed around the recipient BD (Figure 5C).

44. Return the intestines to the body cavity. Instill $2 \mathrm{~mL}$ of 37 ${ }^{\circ} \mathrm{C}$ Ringer's lactate solution into the cavity to flush it. Soak some of the solution up with gauze.

45. Ensure that the intestines are back in their original position before sewing up the parietal peritoneum and skin with 5-0 monocryl.

46. Close the incision in two layers with 5-0 monocryl. Inject $0.5 \mathrm{~mL}$ of $0.5 \%$ bupivacaine around the sewn parietal peritoneum and repeat this once the skin is sewn together.

47. Gently swaddle the recipient rat in a paper towel when transferring to the cage. Allow the animal free access to water and food from the time of awakening. Keep a warm water-circulating blanket under one-half of the cage for 
24-38 h. One rat is designated to one cage during the immediate post-operative period.

\section{Post-operative care}

1. Soak food pellets in water and place them in a Petri dish on the cage floor.

2. Monitor the heart rate, breathing rate, and skin color of the rat.

3. Administer piperacillin on post-operative days 1,2 , and 3. Administer buprenorphine subcutaneously and monitor for any signs of pain such as any behavioral changes, lethargy, ungroomed fur, depression, mutilation, or loss of appetite for the first $72 \mathrm{~h}$.

NOTE: Pain is assessed at least $2 x$ daily for 3 days posttransplantation, then at least $1 \mathrm{x}$ daily onwards.

\section{Representative Results}

While establishing a non-HA anastomosis rat OLT model using a previously described protocol ${ }^{28}$, our team observed $50 \%$ and $37.5 \%$ survival rates at 21 days and 60 days post-operation, respectively. Although high rates of longterm survival without $\mathrm{HA}$ anastomosis have been reported by some groups ${ }^{28}$, these early results highlight the drawbacks of not having arterial inflow. By contrast, the optimized HA reconnection procedure significantly increased long-term survival from $37.5 \%$ to $88.2 \%(p=0.015)$ (Figure 6$)$.
Histological analysis of a representative subset of transplanted animals without HA reconnection (at days 6 and 13 post-operation) showed signs of hypoxic liver injury with centrilobular necrosis (Figure 7). Extensive liver necrosis was associated with tremendously elevated levels of alanine aminotransferase (ALT) and aspartate aminotransferase (AST) in these animals (Figure 7). In contrast, transplanted rats with HA reconnection showed no signs of liver injury, and histological analysis revealed a normal liver parenchyma structure with organized acini, lobules (e.g., central vein and portal triads with hepatic vein), arteries, and bile duct (Figure 7).

Although the mean anhepatic time over the course of 23 separate operations was acceptable (12 $\mathrm{min}$ and 14 $s\left[\begin{array}{lll} \pm & 78 & \mathrm{~s}\end{array}\right]$, it is still possible that survival in the nonHA reconnection model can eventually be improved with increased practice. However, it is worth noting that three of the four animals transplanted without HA reconnection (which were being followed for long-term survival) were euthanized due to distress on days 56,96 , and 111 post-operation. Additionally, histological analysis of the livers revealed reactive changes after hypoxic liver injury including marked bile duct proliferation, periportal fibrosis and inflammation, and distorted liver parenchyma (Supplementary Figure 2). The presence of morphological features of hypoxic liver injury corroborate the findings that $\mathrm{HA}$ reconnection is important for efficient liver perfusion and normal function. 


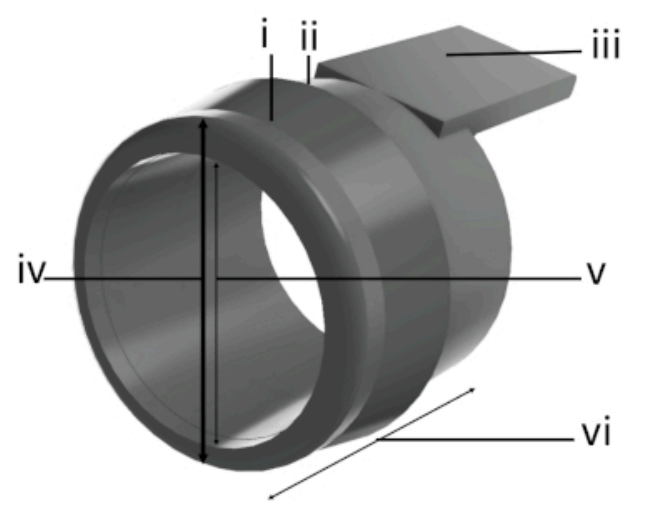

$2.60 \mathrm{~mm}(\mathrm{PV})$

$2.15 \mathrm{~mm}(\mathrm{IHVC})$

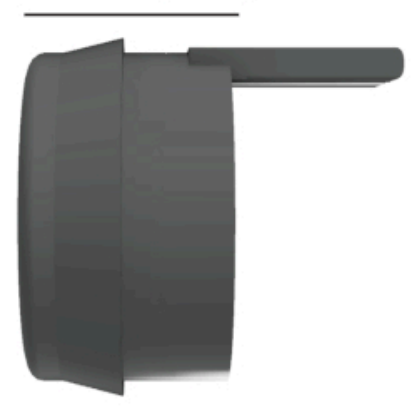

Figure 1: Schematic representation of the 3D-printed cuff design for portal vein and infrahepatic inferior vena cava. The first tie is tightened in the groove (ii) closest to the handle (iii), and the second tie is tightened in the groove (i) furthest away from the handle. The outside diameters are (iv) $2.38 \mathrm{~mm}$ for the portal vein (PV) and $2.15 \mathrm{~mm}$ for the infrahepatic inferior vena cava (IHVC). The inside diameters are (v) $1.74 \mathrm{~mm}$ for the PV and $1.38 \mathrm{~mm}$ for the IHVC. The lengths are (vi) $2.60 \mathrm{~mm}$ for the PV and $2.15 \mathrm{~mm}$ for the IHVC (exact specifications for all 3D-printed materials can be found in Supplementary Materials). Please click here to view a larger version of this figure. 
A
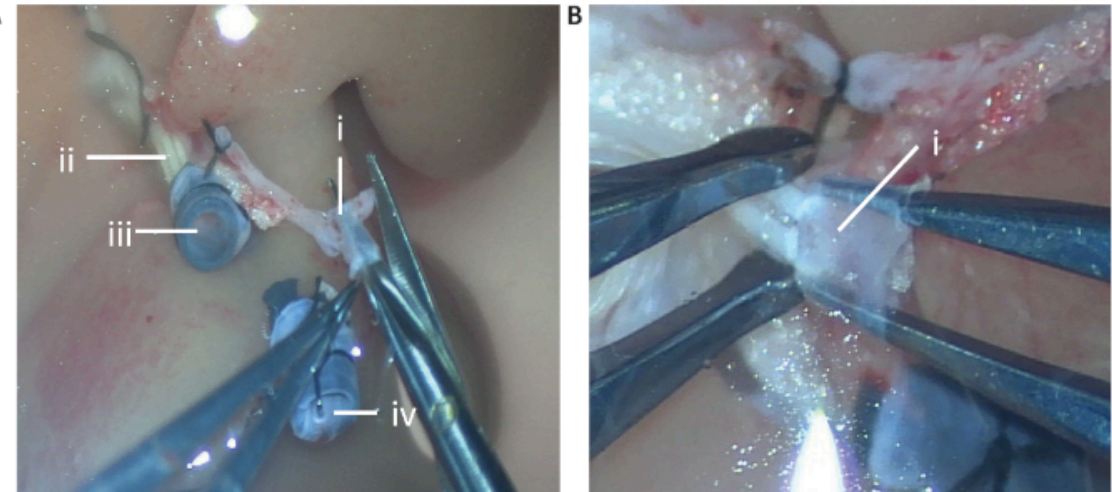

C

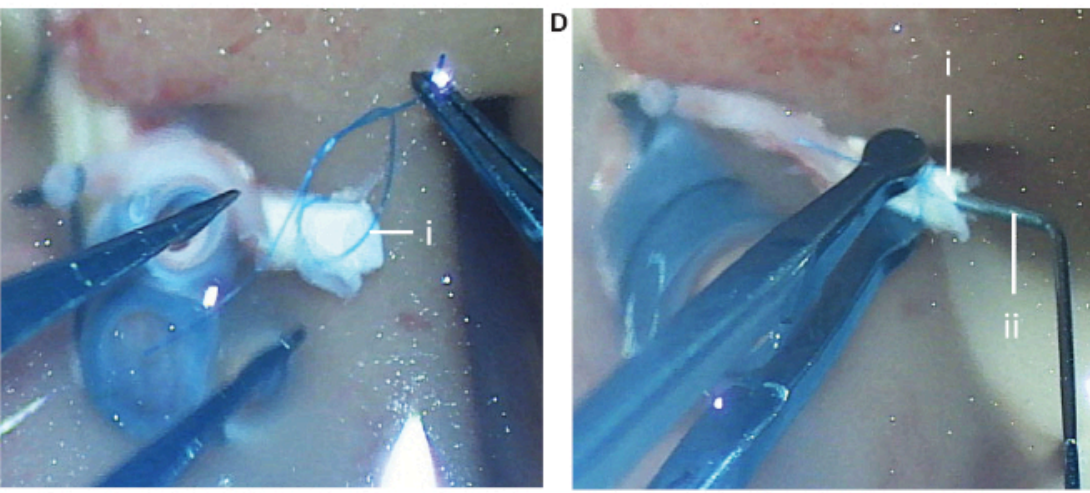

Figure 2: Hepatic artery stent insertion in graft. (A) The opening end of the celiac trunk (i) is widened by cutting the splenic artery to the left gastric artery, which exposes the bifurcation of the common HA. (ii) The BD stent is tied prior to extraction of the donor rat liver. (iii) The PV cuff and (iv) IHVC cuff are inserted and tied by folding the ends of the vessels over the cuff. (B) (i) To insert the HA stent, the exposed common HA is stretched multiple times with forceps. (C) (i) The HA stent is placed securely in the common HA and tied with 8-0 prolene. (D) (i) The HA stent is flushed with (ii) Ringer's lactate solution (BD = bile duct, IHVC = infrahepatic inferior vena cava, $\mathrm{HA}=$ hepatic artery). Please click here to view a larger version of this figure. 

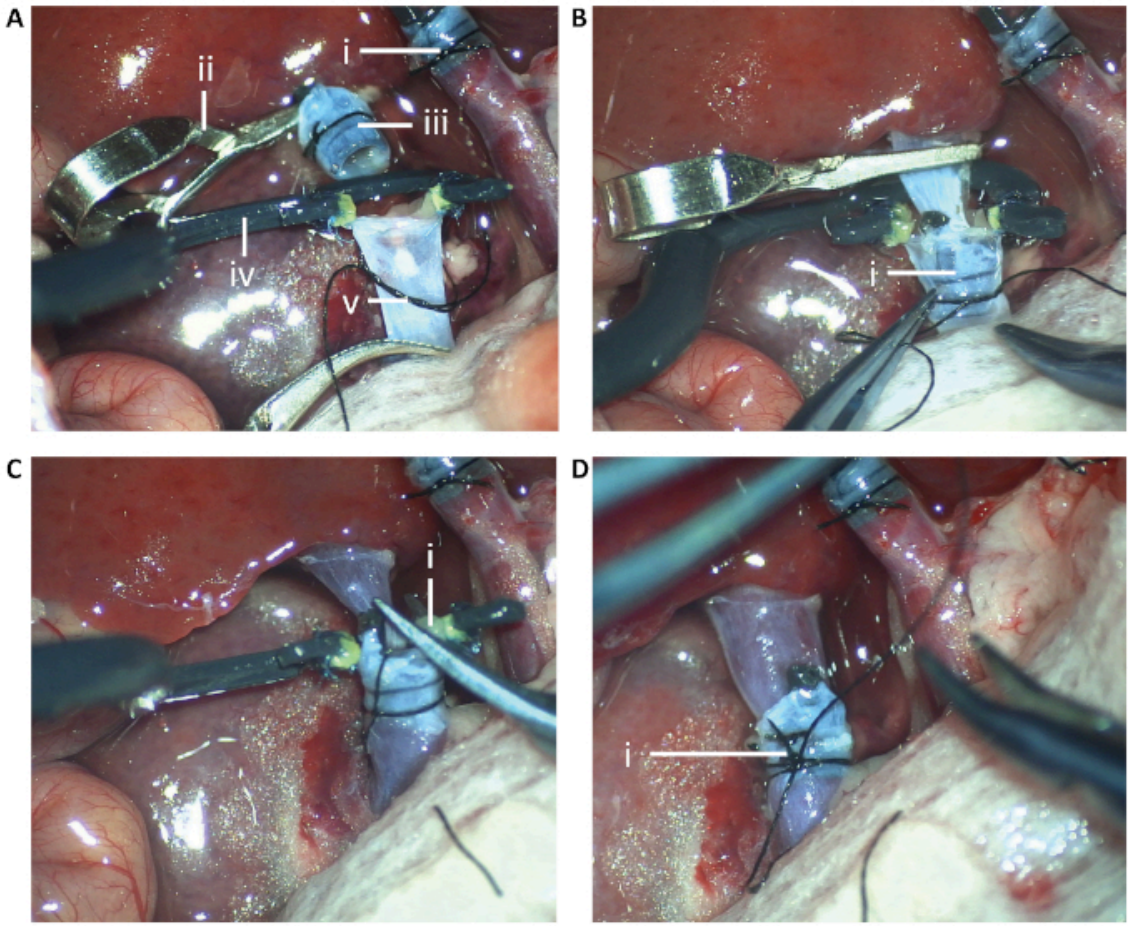

Figure 3: Infrahepatic inferior vena cava connection using 3D-printed holder. (A) The (i) PV is connected using the same technique as the IHVC connection. The graft is (ii) clamped above the (iii) IHVC cuff. The recipient IHVC opening is (iv) sutured at the sides of the opening to a 3D-printed holder to keep it stretched open. A loose (v) 7-0 silk is tied around the recipient IHVC. (B) The cuff of the graft IHVC is (i) inserted within the recipient IHVC. The loose tie is now tightened. (C) The clamp is removed, and (i) the 3D printed holder is detached with scissors. (D) An additional (i) 7-0 silk is tied around the connection if not secure, but typically one tie is sufficient (PV = portal vein, IHVC = infrahepatic inferior vena cava). Please click here to view a larger version of this figure. 

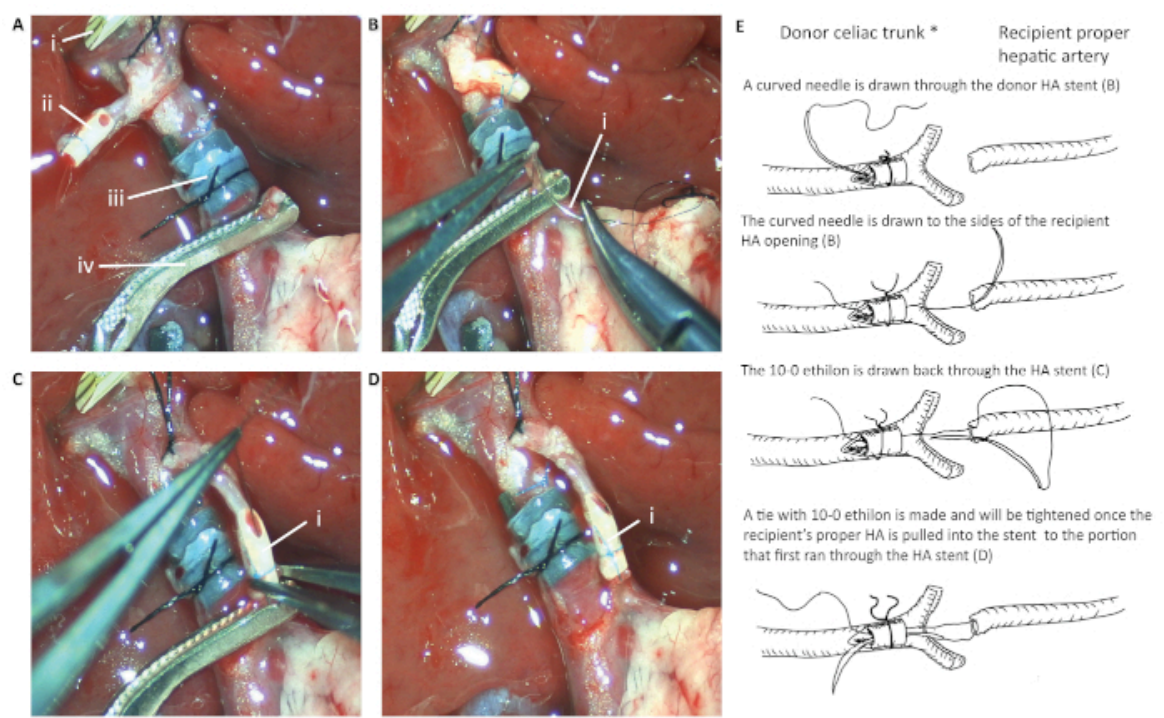

Figure 4: Microvascular sleeve connection of the hepatic artery. (A) (i) The BD stent is not connected to the recipient. (ii) The HA stent is placed in the graft, which is linked to the (iv) recipient's proper HA. (iii) The PV is connected. (B) 10-0 ethilon with a (i) curved needle is drawn through the HA stent to the sides of the recipient HA opening end. (C) The 10-0 ethilon is drawn back through the HA stent; therefore, the recipient's proper HA is pulled through the stent like a sleeve. (D) (i) A tie with 10-0 ethilon is made once the recipient's proper HA is pulled into the stent to the portion that first runs through the HA stent. (E) Shown here is a schematic of the HA anastomosis described in (B), (C), and (D) (BD = bile duct, HA = hepatic artery, $\mathrm{PV}=$ portal vein). ${ }^{*}$ The opening end of the celiac trunk is widened by cutting the splenic artery to the left gastric artery. Please click here to view a larger version of this figure. 
A

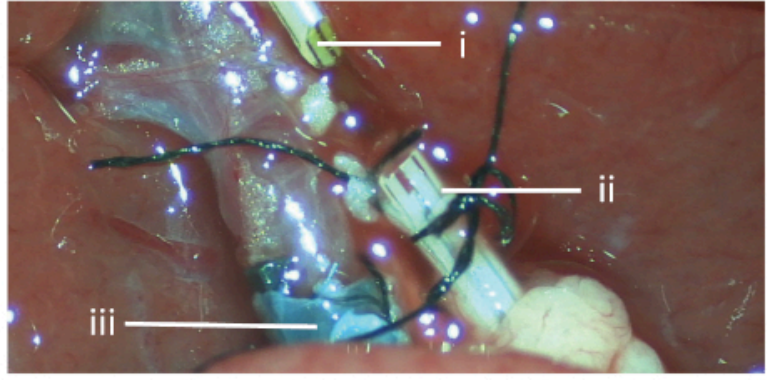

B
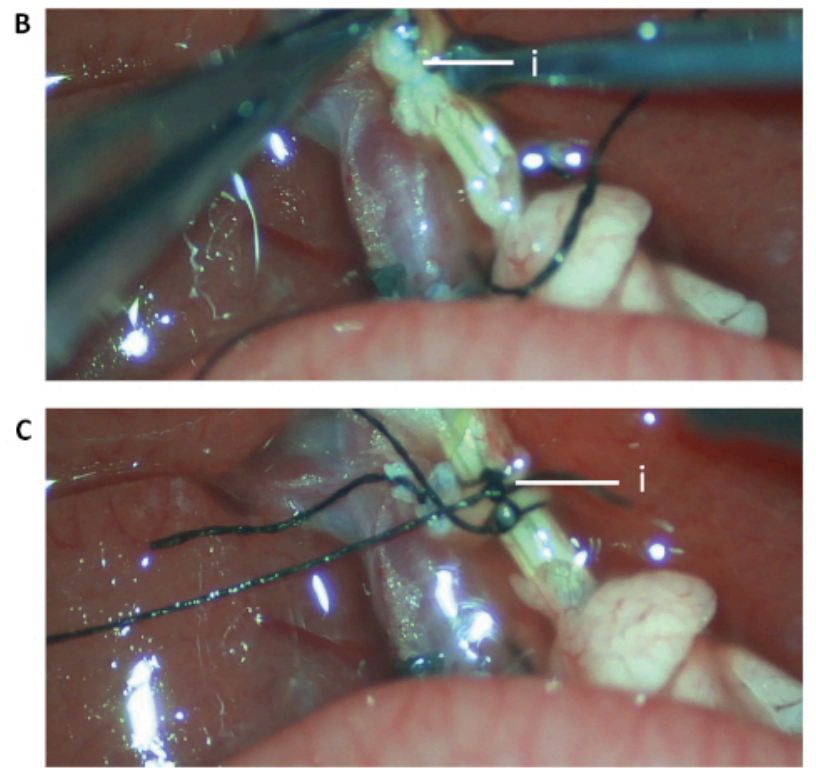

Figure 5: Bile duct connection using two stents. (A) (i) Graft BD stent is inserted into the recipient BD with the aid of the (ii) stent loosely tied at the opening of the recipient's BD. (iii) The PV is linked before the BD connection, which is located behind the BD. (B) The stent at the end of the recipient's BD is removed and used as a widened opening to (i) insert the BD stent tied to the graft. (C) The tie that is loosely securing the recipient stent is now used to tie the connection, and (i) another 7-0 silk is used to firmly keep the stent in place to avoid slipping or twisting of the stent. Please click here to view a larger version of this figure. 


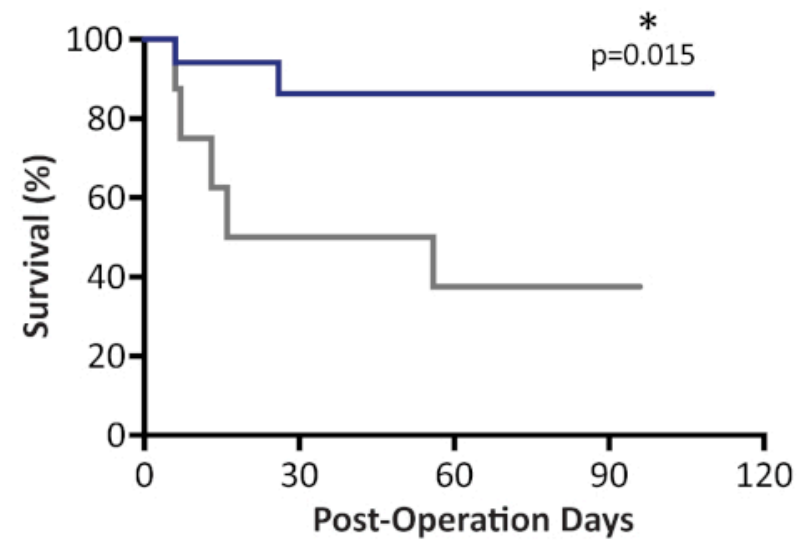

- With HA Reconnection

- Without HA Reconnection

Figure 6: Transplant percent survival. Orthotopic rat liver transplantation without HA reconnection $(n=8)$ and with HA reconnection $(n=17)$. Animals are closely followed post-transplantation for signs of liver failure and/or infection for at least 60 days. Rats that showed no complications after surgery were considered survivors $\left({ }^{*} p=0.015\right.$, as calculated by KaplanMeier estimation [long rank test]). Please click here to view a larger version of this figure. 


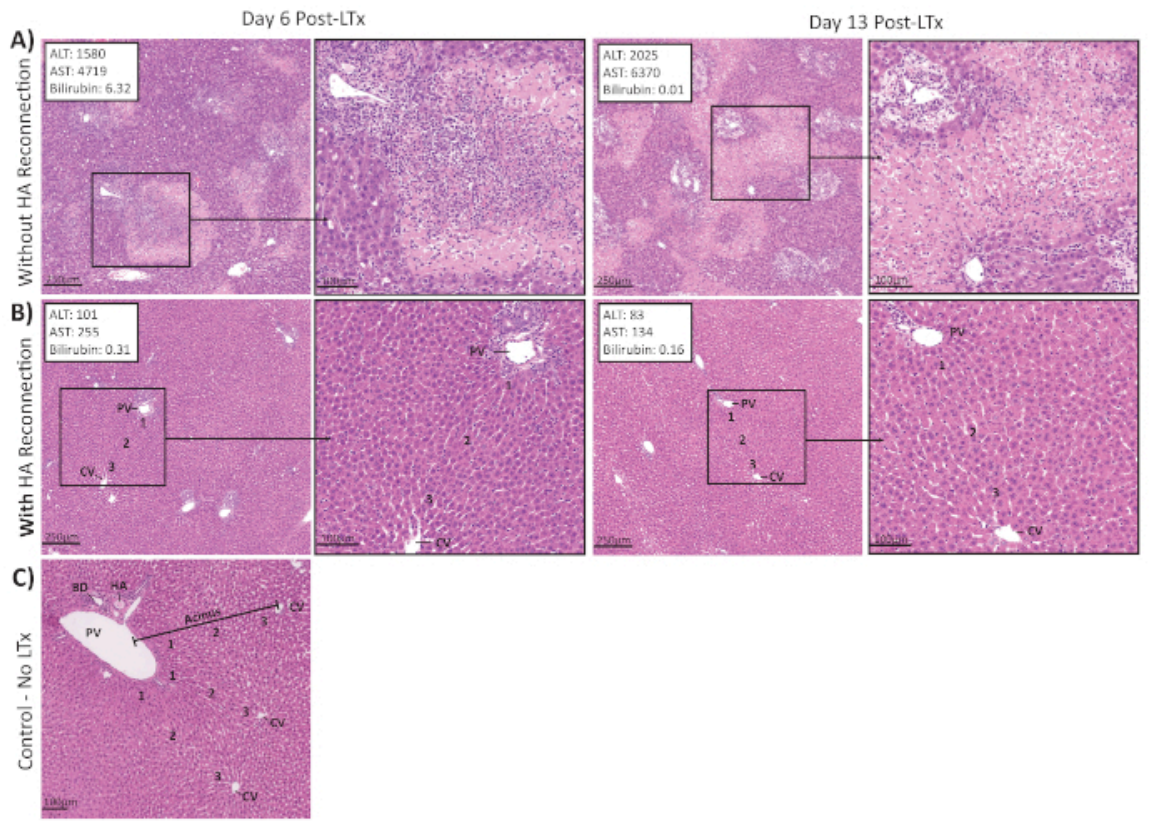

Figure 7: Liver histopathological assessment. Representative hematoxylin and eosin-stained sections in animals (A) without and (B) with hepatic artery (HA) reconnection at days 6 and 13 after liver transplant (LTx). (C) Normal liver parenchyma showing portal triad (portal vein, artery, and bile duct), lobules including central vein, and acini. Hepatocytes next to the portal triad are zone 1 hepatocytes; hepatocytes next to the central vein within lobules are zone 3 hepatocytes; and hepatocytes between zones 1 and 3 are zone 2 hepatocytes (ALT = alanine aminotransferase, AST = aspartate aminotransferase, $\mathrm{CV}=$ central vein). Please click here to view a larger version of this figure. 


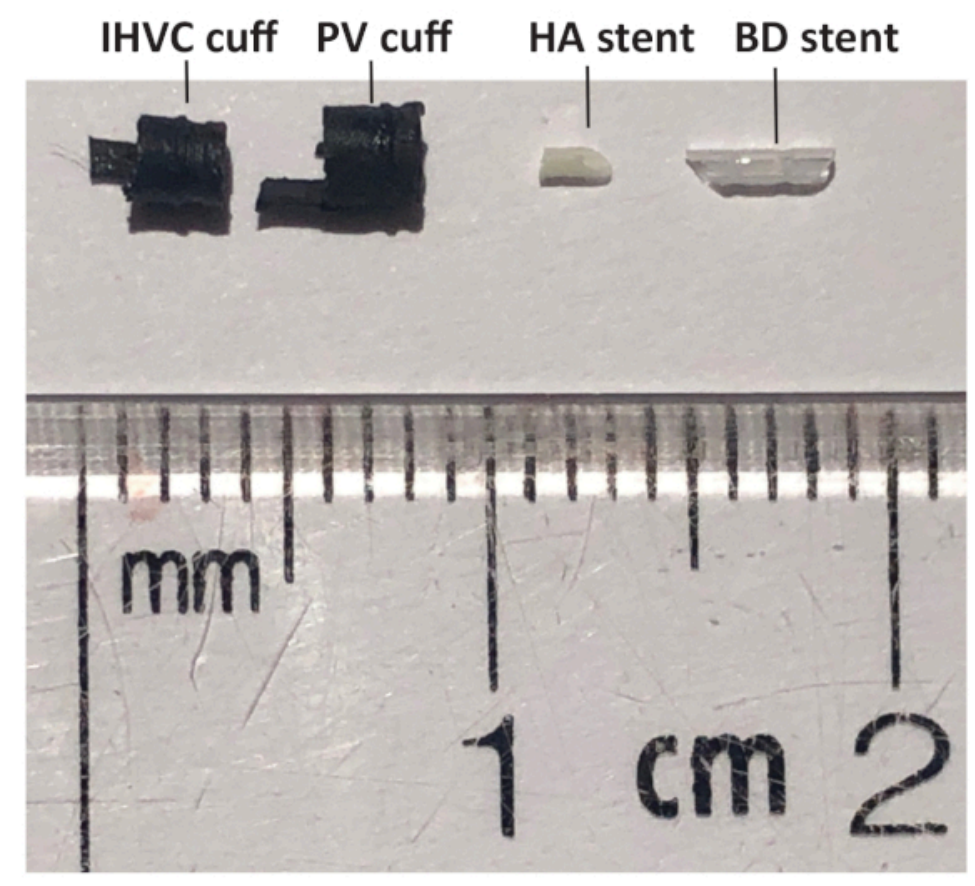

Supplementary Figure 1: Stent and cuff dimensions. Please click here to view a larger version of this figure.

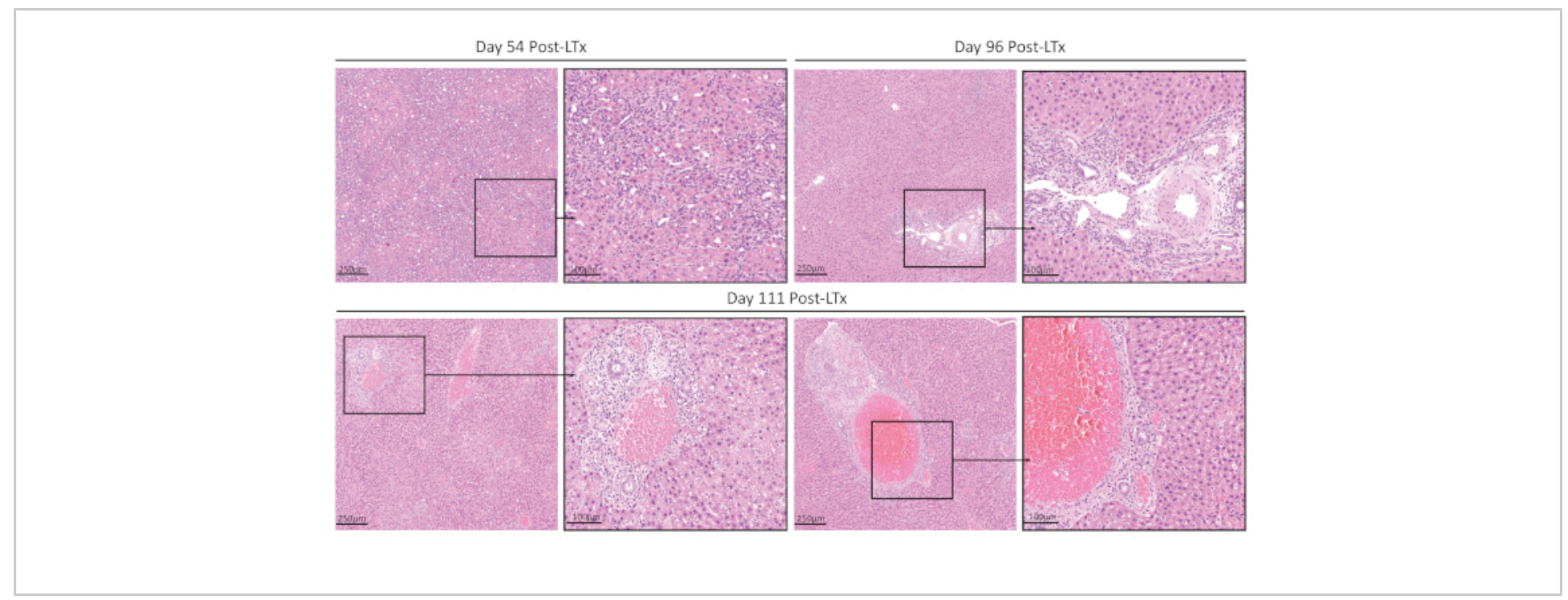

\section{Supplementary Figure 2: Liver histopathological assessment showing disruption of liver parenchyma.}

Representative hematoxylin and eosin-stained sections in animals without HA reconnection at days 54, 96, and 111 after LTX. Please click here to view a larger version of this figure. 
Supplementary Material 1: Porta cuff $200 \mathrm{~g}$ - support 2.0. Please click here to view this file (Right click to download).

Supplementary Material 2: Cava cuff $200 \mathrm{~g}$ - support 2.0. Please click here to view this file (Right click to download).

Supplementary Material 3: Liver retractor 200g. Please click here to view this file (Right click to download).

Supplementary Material 4: Back holder - 1.2. Please click here to view this file (Right click to download).

Supplementary Material 5: Cava $150 \mathrm{~g}-2.1$. Please click here to view this file (Right click to download).

Supplementary Material 6: Porta 1.4.1. Please click here to view this file (Right click to download).

Supplementary Material 7: Holder arm McGil. Please click here to view this file (Right click to download).

Supplementary Material 8: Holder mini arm LAB. Please click here to view this file (Right click to download).

Supplementary Material 9: Holder and arm soft part 1.3. Please click here to view this file (Right click to download).

\section{Supplementary Material 10: Holder base - 3.1. Please click} here to view this file (Right click to download).

\section{Discussion}

Small animal liver transplantation models are important for understanding transplant immunity and identifying novel therapeutic strategies ${ }^{32}$. The ideal small animal liver transplantation model replicates all steps of the human procedure, including arterial anastomosis. It can be challenging to interpret results from the rat OLT model, as most versions do not incorporate an HA anastomosis step, which leads to higher rates of complications and morbidity 42 .
Some reconstruction procedures have used the renal artery, which requires removal of the kidney ${ }^{27}$. This protocol avoids organ removal, as it is beyond what occurs in the human procedure.

Arterial reconstructions can also be performed by manipulating the rat aorta ${ }^{31}$. However, these methods require extensive dissection and clamping of the aorta. If the clamp time is prolonged, then the recipient rat will have poor outcomes related to distal ischemia ${ }^{43}$. In humans, a LT surgical technique involves the ligation and division of the recipient gastroduodenal artery (GDA). However, the physiological and anatomical features of rodents make transplantation using this technique more physiologically challenging and may lead to complications (i.e., necrosis of the pancreas and bile duct ${ }^{35}$ and bile leakage $\left.{ }^{44}\right)$. The arterial reconnection in this protocol is intended to circumvent this challenge, maintain duct blood flow, and improve recipient outcome.

The use of a sleeve and stenting technique for reconstruction of the rat HA has been described previously ${ }^{27}$. In this technique, a stent is used as a guide, and the artery is reconstructed from the donor celiac trunk to the recipient common HA. The recipient common HA is then dissected out, and the recipient GDA is tied off ${ }^{27}$. As a result, the blood supply to the lower part of the recipient BD and head of the pancreas may become compromised. It is believed that the collateral circulation to this area often provides inadequate blood flow to the bile duct. For example, this protocol testclamps the recipient GDA first with a microvascular clip, then divides the recipient BD. With the GDA clamped, the divided BD does not bleed. After removing the GDA clamp, brisk bleeding from the BD is observed. This protocol, which maintains good flow to the divided recipient bile duct, protects 
the physiology of the recipient liver tissue by providing adequate liver blood perfusion and preventing post-OLT hypoxic liver injury.

On the donor side, the HA stent is inserted into the celiac trunk during the graft preparation with ease by creating a patch from the celiac trunk, left gastric artery, and splenic artery. The stent can be inserted through the broad opening, which is less difficult than trying to insert the stent into the celiac trunk alone. It has been found that $24 \mathrm{G}$ is an ideal size to use for the HA stent. The length of the stent should be 1.0-1.5 mm long, because it acts as an open gate to allow the recipient's proper $\mathrm{HA}$ to be readily pulled into the donor's common HA. With careful attention to where the $10-0$ ethilon suture is placed, blood flowing through this connection will never touch the stent directly, and the recipient's proper HA will shield it from the inside, reducing the risk of complications. Importantly, the donor's HA is never clamped in order to avoid vasospasm. The success of arterial reconstruction is evaluated by leaving the donor GDA open. Successful anastomosis results in good blood flow from the donor GDA once reconstruction is completed.

In this protocol, similar to others, SHVC reconnection is the slowest step and ultimately dictates the duration of the anhepatic phase. As the duration of the anhepatic time increases, the risk ischemic injury and liver dysfunction increases $^{45}$. Another critical component of OLT rat models is the sizes of the graft, stents, and cuffs. If the graft is too small, the graft may twist or flip, obstructing the vascular connections. The size of the stents and cuffs may require adjustments according to the age, sex, weight, and strain of the rat. The size of the cuffs used here was chosen as previously described ${ }^{28}$, and one cuff size that controlled for rat size was used. There were no signs of distress or complications (i.e., liver congestion, edema, ascites, or splenomegaly) during the follow-up period (to date: median = 133 days post-operation, minimum = 115 days post-op, maximum $=161$ days post-op). Further studies to determine the suitable size of $\mathrm{PV}$ and IHVC for various rat strains accounting for both age and sex are warranted.

This modified rat OLT protocol uses 3D-printed cuffs for the PV and IHVC, as described previously ${ }^{39,40}$. Existing methods for connecting the PV and IHVC include a microsuture technique ${ }^{32}$, cuff technique ${ }^{46}$, and microsuturetemporary splint technique 47 . The 3D-printed cuff technique was chosen, since it allows the size of the cuff to be standardized according to rat strain and is easy to prepare and use. Large quantities of cuffs with the same dimensions can be printed at once. The outer surface of the cuff has two grooves to aid with securing ties and prevent slipping. A tail is also incorporated into the cuff design to allow for easier manipulation of the cuff. Overall, it is believed that incorporating 3D-printed cuffs leads to high success rates and reproducibility of the OLT procedure by shortening anhepatic time. It is determined that this technique also shortens the surgical learning curve.

In conclusion, the described protocol established a model that is more similar to human liver transplantation by incorporating an arterial reconnection step. This protocol can be adapted to study many immunologic and surgical aspects of liver transplantation and can serve as a model to test novel therapeutic interventions relevant to transplantation.

\section{Disclosures}

The authors have nothing to disclose.

\section{Acknowledgments}


This research was funded through funds from the Multi-Organ transplant program at the UHN and support from the Toronto General and Toronto Western Foundation.

\section{References}

1. Global Observatory on Donation and Transplantation. Organ Donation and Transplantation Activities. http:// www.transplant-observatory.org/download/2016-activitydata-report/ (2016).

2. Asrani, S. K., Devarbhavi, H., Eaton, J., Kamath, P. S. Burden of liver diseases in the world. Journal of Hepatology. 70 (1), 151-171 (2019).

3. Dopazo, C. et al. Analysis of adult 20-year survivors after liver transplantation. Hepatology International. 9 (3), 461-470 (2015).

4. Schoening, W. N. et al. Twenty-year longitudinal followup after orthotopic liver transplantation: a single-center experience of 313 consecutive cases. American Journal of Transplantation. 13 (9), 2384-2394 (2013).

5. Pischke, S. et al. Factors associated with long-term survival after liver transplantation: A retrospective cohort study. World Journal of Hepatology. 9 (8), 427-435 (2017).

6. Hamdani, S. et al. Delayed and short course of rapamycin prevents organ rejection after allogeneic liver transplantation in rats. World Journal of Gastroenterology. 23 (38), 6962-6972 (2017).

7. Endo, K. et al. Pretransplant replacement of donor liver grafts with recipient Kupffer cells attenuates liver graft rejection in rats. Journal of Gastroenterology and Hepatology. 30 (5), 944-951 (2015).
8. Zhao, Z. et al. IL-34 Inhibits Acute Rejection of Rat Liver Transplantation by Inducing Kupffer Cell M2 Polarization. Transplantation. 102 (6), e265-e274 (2018).

9. Nagakawa, Y. et al. Over-expression of AIF-1 in liver allografts and peripheral blood correlates with acute rejection after transplantation in rats. American Journal of Transplantation. 4 (12), 1949-1957 (2004).

10. Gao, L. H., Zeng, L. X., Chen, H. M., Wan, R. $\mathrm{H}$. Cytomegalovirus infection accelerates the process of chronic rejection in rat liver transplantation. Transplantation Proceedings. 45 (6), 2536-2538 (2013).

11. $\mathrm{Wu}, \mathrm{Y}$. et al. Effects of combined genes of CTLA4Ig and IDO in post-liver transplantation immune tolerance of rats. Annals of Hepatology. 15 (5), 729-737 (2016).

12. He, X. S. et al. Influence of warm ischemia injury on hepatic functional status and survival of liver graft in rats. Hepatobiliary and Pancreatic Diseases International. 2 (4), 504-508 (2003)

13. Tamura, A. et al. Combination effect of tacrolimus and FTY720 in liver transplantation in rats. Transplantation Proceedings. 31 (7), 2785-2786 (1999).

14. Wang, Z. et al. RhGH attenuates ischemia injury of intrahepatic bile ducts relating to liver transplantation. Journal of Surgical Research. 171 (1), 300-310 (2011).

15. Jiang, J. W. et al. Chronic bile duct hyperplasia is a chronic graft dysfunction following liver transplantation. World Journal of Gastroenterology. 18 (10), 1038-1047 (2012)

16. Tang, Y. et al. S-Adenosylmethionine attenuates bile duct early warm ischemia reperfusion injury after rat liver transplantation. Molecular Immunology. 95, 83-90 (2018). 
17. Nosaka, T., Bowers, J. L., Cay, O., Clouse, M. E. Biliary complications after orthotopic liver transplantation in rats. Surgery Today. 29 (9), 963-965 (1999).

18. Howden, B., Jablonski, P., Grossman, H., Marshall, V. C. The importance of the hepatic artery in rat liver transplantation. Transplantation. 47 (3), 428-431 (1989).

19. Post, S. et al. The impact of arterialization on hepatic microcirculation and leukocyte accumulation after liver transplantation in the rat. Transplantation. 54 (5), 789-794 (1992).

20. Hori, T. et al. Impact of hepatic arterial reconstruction on orthotopic liver transplantation in the rat. Journal of Investigative Surgery. 25 (4), 242-252 (2012).

21. Zhou, S. et al. New method of stent-facilitated arterial reconstruction for orthotopic mouse liver transplantation. Journal of Surgical Research. 187 (1), 297-301 (2014).

22. Noack, K., Bronk, S. F., Kato, A., Gores, G. J. The greater vulnerability of bile duct cells to reoxygenation injury than to anoxia. Implications for the pathogenesis of biliary strictures after liver transplantation. Transplantation. 56 (3), 495-500 (1993).

23. Imamura, H., Rocheleau, B., Cote, J., Huet, P. M. Longterm consequence of rat orthotopic liver transplantation with and without hepatic arterial reconstruction: a clinical, pathological, and hemodynamic study. Hepatology. 26 (1), 198-205 (1997).

24. Reck, T. et al. Impact of arterialization on hepatic oxygen supply, tissue energy phosphates, and outcome after liver transplantation in the rat. Transplantation. 62 (5), 582-587, (1996).

25. Zhao, D., Wheatley, A. M. Orthotopic liver transplantation in the rat: comparison of models with and without rearterialization of the graft. European Surgical Research. 25 (5), 294-302 (1993).

26. Chaland, P. et al. Orthotopic liver transplantation with hepatic artery anastomoses. Hemodynamics and response to hemorrhage in conscious rats. Transplantation. 49 (4), 675-678 (1990).

27. Liu, X., He, C., Huang, T., Gu, J. Development of a New Technique for Reconstruction of Hepatic Artery during Liver Transplantation in Sprague-Dawley Rat. PLoS One. 10 (12), e0145662 (2015).

28. Oldani, G., Lacotte, S., Morel, P., Mentha, G., Toso, C. Orthotopic liver transplantation in rats. Journal of Visualized Experiments. 10.3791/4143 (65) (2012).

29. Lee, S., Charters, A. C., Chandler, J. G., Orloff, M. J. A technique for orthotopic liver transplantation in the rat. Transplantation. 16 (6), 664-669 (1973).

30. Kamada, N., Calne, R. Y. Orthotopic liver transplantation in the rat. Technique using cuff for portal vein anastomosis and biliary drainage. Transplantation. 28 (1), 47-50 (1979).

31. Kashfi, A. et al. A review of various techniques of orthotopic liver transplantation in the rat. Transplantation Proceedings. 37 (1), 185-188 (2005).

32. Chong, A. S., Alegre, M. L., Miller, M. L., Fairchild, R. L. Lessons and limits of mouse models. Cold Spring Harbor Perspectives in Medicine. 3 (12), a015495 (2013).

33. Hasuike, Y. et al. A simple method for orthotopic liver transplantation with arterial reconstruction in rats. Transplantation. 45 (4), 830-832 (1988).

34. Hickman, R., Engelbrecht, G. H., Duminy, F. J. A technique for liver transplantation in the rat. Transplantation. 48 (6), 1080 (1989). 
35. Steffen, R., Ferguson, D. M., Krom, R. A. A new method for orthotopic rat liver transplantation with arterial cuff anastomosis to the recipient common hepatic artery. Transplantation. 48 (1), 166-168 (1989).

36. Shi, Y. et al. Magnetic ring anastomosis of suprahepatic vena cava: novel technique for liver transplantation in rat. Transplant International. 28 (1), 89-94 (2015).

37. Dippe, B. E. et al. An improved model for rat liver transplantation including arterial reconstruction and simplified microvascular suture techniques. Journal of Investigative Surgery. 5 (4), 361-373 (1992).

38. Kobayashi, E., Kamada, N., Goto, S., Miyata, M. Protocol for the technique of orthotopic liver transplantation in the rat. Microsurgery. 14 (8), 541-546 (1993).

39. Oldani, G. et al. Efficient nonarterialized mouse liver transplantation using 3-dimensional-printed instruments. Liver Transplation. 22 (12), 1688-1696 (2016).

40. Oldani, G. et al. Manufacturing devices and instruments for easier rat liver transplantation. Journal of Visualized Experiments. 10.3791/50380 (75), e50380 (2013).

41. Li, J. et al. Modified sleeve anastomosis for reconstruction of the hepatic artery in rat liver transplantation. Microsurgery. 22 (2), 62-68 (2002).

42. Li, G. L. et al. High incidence of biliary complications in rat liver transplantation: can we avoid it? World Journal of Gastroenterology. 17 (26), 3140-3144 (2011).

43. Zammert, M., Gelman, S. The pathophysiology of aortic cross-clamping. Best Practice and Research: Clinical Anaesthesiology. 30 (3), 257-269 (2016).

44. Gao, W., Lemasters, J. J., Thurman, R. G. Development of a new method for hepatic rearterialization in rat orthotopic liver transplantation. Reduction of liver injury and improvement of surgical outcome by arterialization. Transplantation. 56 (1), 19-24 (1993).

45. Ijtsma, A. J. et al. The clinical relevance of the anhepatic phase during liver transplantation. Liver Transplation. 15 (9), 1050-1055 (2009).

46. Miyata, M., Fischer, J. H., Fuhs, M., Isselhard, W., Kasai, Y. A simple method for orthotopic liver transplantation in the rat. Cuff technique for three vascular anastomoses. Transplantation. 30 (5), 335-338 (1980).

47. Marni, A., Ferrero, M. E. A four-technique comparative study of orthotopic liver transplantation in the rat. American Journal of Surgery. 156 (3 Pt 1), 209-213 (1988). 